2

6

\title{
Water partitioning between bridgmanite and postperovskite in the lowermost mantle
}

\author{
Joshua P Townsend ${ }^{\mathrm{a}, *}$, Jun Tsuchiya ${ }^{\mathrm{b}}$, Craig R Bina ${ }^{\mathrm{a}}$, Steven D Jacobsen ${ }^{\mathrm{a}}$ \\ ${ }^{a}$ Department of Earth and Planetary Sciences, Northwestern University, Evanston, IL \\ 60208, USA \\ ${ }^{b}$ Geodynamics Research Center, Ehime University, Matsuyama, Ehime 790, Japan
}

7 Abstract

The lowermost mantle appears to contain geochemically primitive reservoirs of volatile components including water, as evidenced by certain ocean island basalts [17]. We used ab-initio lattice dynamics to calculate the water partition coefficient between bridgmanite and postperovskite using quasiharmonic free energies to determine how water is distributed between nominally anhydrous minerals in the $\mathrm{D}^{\prime \prime}$ region. In the absence of aluminum, hydrogen was incorporated into both phases by a simple substitution of $\mathrm{Mg}^{2+} \Leftrightarrow$ $2 \mathrm{H}^{+}$, and we found that water favors bridgmanite over postperovskite by a factor of about 5:1 at conditions where an average mantle geotherm intersects the phase boundary. In the Al-bearing system, hydrogen and aluminum were coupled as $\mathrm{Si}^{4+} \Leftrightarrow \mathrm{Al}^{3+}+\mathrm{H}^{+}$defects into both phases, and we found that water favors postperovskite over bridgmanite in the Al-bearing system by a factor of about 3:1 at ambient mantle conditions, and by about 8:1 at colder slab conditions. Our results indicate that aluminum controls the partitioning of water between bridgmanite and postperovskite, and that aluminous postperovksite may be a potential host for primordial water in the lowermost region of the mantle. The strong partitioning of water into aluminous

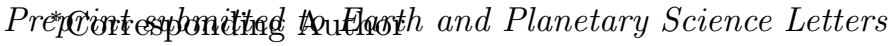

June 29, 2016

Email address: joshua@earth.northwestern.edu (Joshua P Townsend )

(C) 2016. This manuscript version is made available under the Elsevier user license http://www.elsevier.com/open-access/userlicense/1.0/ 
postperovskite over bridgmanite provides a potential mechanism for dehydration melting in the lowermost mantle that could be a source for ocean island basalts in regions of upwelling.

8 Keywords: Nominally anhydrous minerals, Water partitioning, Density functional theory

\section{Introduction}

A comprehensive geochemical and geophysical description of the evolution and state of the lowermost mantle, or $\mathrm{D}^{\prime \prime}$ region, remains a challenging problem more than 60 years after its discovery [8]. The $\mathrm{D}^{\prime \prime}$ region is complex but is typically characterized by anti-correlated bulk and shear wave velocities, laterally varying thickness between 0 to $>300 \mathrm{~km}$, complicated seismic anisotropy structure, and large regions of generally low shear wave velocities $[18,29,10]$. These features have been attributed to chemical heterogeneity [49], phase transformations [31], and partial melting [12]. More recently, geochemical and geodynamical evidence suggests the existence of primordial un-degassed, volatile-rich reservoirs in the lowermost mantle [17, 50, 15]. Yet after more than a decade of work on the bridgmanite-postperovskite (brg-ppv) phase transition, the distribution of volatile elements and melt generation in the lowermost mantle remains poorly understood $[13,11]$.

The effects of major element chemistry on the brg-ppv transition to date have focused mostly on iron and aluminum. For pure $\mathrm{MgSiO}_{3}$ the brg-ppv phase transformation occurs at approximately $125 \mathrm{GPa}$ and $2500 \mathrm{~K}$, corresponding to about $2700 \mathrm{~km}$ depth; about $300 \mathrm{~km}$ above the core-mantle boundary (CMB) along a mantle geotherm. The Clapeyron slope of the 
phase boundary has been estimated to be $4-14 \mathrm{MPa} / \mathrm{K}$, and while well defined only for univariant systems, the effective Clapeyron slope of the $\mathrm{Al}$ and Fe-bearing systems indicates that it is relatively insensitive to composition [31, 10, 48, 34]. However, for expected mantle compositions including aluminum, there exists a two-phase region of coexisting brg and ppv which reduces the velocity gradient and slightly decreases the transition depth in the case of aluminum, but not appreciably in the case of iron $[1,44,20]$. Experiments on iron- and aluminum-bearing compositions indicate that a pyrolitic $\mathrm{D}^{\prime \prime}$ might not contain any ppv, while for MORB compositions the transition depth may lie several hundred kilometers above the CMB [16].

Despite the focus on the effects of major element composition, the influence of geophysically important trace impurities such as water on the brgppv transition have not been investigated. Hernández et al. [19] estimated a ringwoodite:bridgmanite (rw:brg) water partition coefficient of 1:0.1 at 24 GPa and $1500 \mathrm{~K}$ via ab-intio density functional theory (DFT) techniques. Panero et al. [37] synthesized bridgmanite in the diamond-anvil cell from naturally-occurring enstatite containing 2065 ppm $\mathrm{H}_{2} \mathrm{O}$ at 25-65 $\mathrm{GPa}$ and $1600-2000 \mathrm{~K}$, finding that likely no more than 10 ppm $\mathrm{H}_{2} \mathrm{O}$ entered the brg phase. Complementary first-principles calculations under static $(0 \mathrm{~K})$ conditions indicated that Mg-bridgmanite can accommodate a maximum of 30-40 ppm $\mathrm{H}_{2} \mathrm{O}$ at conditions throughout the lower mantle. Although no experimental work has been performed on water partitioning between ppv and brg, Townsend et al. [42] described a hydrous post-perovskite structure (hppv) using DFT methods stable under $\mathrm{D}^{\prime \prime}$ pressures. The hppv structure, charge balanced by $\mathrm{Mg}$-site vacancies $\left(\mathrm{Mg}_{1-x} \mathrm{H}_{2 x} \mathrm{SiO}_{3}\right)$, exhibits bulk and 
shear moduli that are reduced by about 7-10 GPa/wt\% $\mathrm{H}_{2} \mathrm{O}$ compared with dry ppv at $\mathrm{D}^{\prime \prime}$ pressures.

To investigate the water partition coefficient between brg and ppv in the lowermost mantle, we used first-principles calculations of total energy and ab-initio lattice dynamics simulations to compute the quasi-harmonic free energies of for two varieties of hydrogen defects, with and without aluminum, in bridgmanite and postperovskite. For aluminum-bearing hydrous compositions we find that ppv is stabilized relative to brg under static conditions, and that the water partition coefficient of ppv relative to brg is 3.2:1 along an ambient mantle geotherm, and 8.1:1 along a slab geotherm. Our results demonstrate that aluminous postperovskite may store significantly more water than aluminous bridgmanite, and suggest that $\mathrm{D}^{\prime \prime}$ may host primordial water even in the absence of dense-hydrous-magnesium-silicates such as phase $\mathrm{H}$ [33]. The bulk sound velocity impedance contrast across the brg-ppv transition and the ppv velocity gradient are more sensitive to the presence of water than the velocities themselves, which suggests that velocity perturbations alone may not be sufficient to investigate the hydration state of the lower mantle. In regions of the lowermost mantle where upwelling primordial hydrous postperovskite transforms to bridgmanite, water would be liberated due to the large difference in partitioning between these phases, and might generate melts at the base of the mantle by dehydration reactions, analogous to dehydration melting reactions across the 410 - and $660-\mathrm{km}$ discontinuities of the mantle transition zone [5, 35, 21, 39]. 

87 1):

\section{Methods}

\subsection{Partition coefficient}

We computed the water partition coefficient between brg and ppv in two systems. In the aluminum-free system hydrogen is incorporated into both phases by $\mathrm{Mg}^{2+} \Leftrightarrow 2 \mathrm{H}^{+}$(hereafter called $\mathrm{Mg}-2 \mathrm{H}$ mechanism). In the aluminum-bearing system hydrogen is incorporated into both phases by a coupled substitution $\mathrm{Si}^{4+} \Leftrightarrow \mathrm{Al}^{3+}+\mathrm{H}^{+}$(hereafter called $\mathrm{Si}-\mathrm{AlH}$ mechanism). Following the procedure of Hernández et al. [19], the water partition coefficient $D$ between brg and ppv for a particular defect type $Y$ is controlled by the Gibbs free-energy of formation of the defect in each phase (see Appendix

$$
{ }^{Y} D_{b r g}^{p p v}=\frac{e^{G_{b r g}^{f} / k_{B} T}+1}{e^{G_{p p v}^{f} / k_{B} T}+1} \approx e^{\Delta G_{b r g-p p v}^{f} / k_{B} T}
$$

where ${ }^{Y} D_{b r g}^{p p v}$ is the partition coefficient of defect $Y$ in ppv relative to brg, $G^{f}$ is the Gibbs free-energy of formation of a $Y$ defect in brg or ppv, $k_{B}$ and $T$ are Boltzmann's constant and temperature, respectively. Therefore, in order to compute the partition coefficient, the Gibbs free energies for all phases with and without defects is required. The above formulation assumes that defects are partitioning between two solid phases in the absence of any fluids. As with previous work (cf. [19]), the results do not represent water storage capacity which is defined as the amount of water in a nominally anhydrous mineral in equilibrium with a hydrous melt [26].

\subsection{Computational Technique}

First-principles calculations of total energy for each structure were carried out with the Quantum ESPRESSO package, an implementation of density 
functional theory with plane wave basis sets $[22,27,14]$. The exchangecorrelation energy was computed using the Perdew-Ernzerhof-Burke generalized gradient approximation [38], and the k-space mesh was generated by the Monkhorst-Pack scheme [30]. Norm-conserving pseudopotentials were used to describe $\mathrm{H}, \mathrm{O}$, and $\mathrm{Si}[43]$, and the $\mathrm{Mg}$ pseudopotential was generated by the method of von Barth and Car [4]. These pseudopotentials have been used in numerous investigations of both hydrous and anhydrous mantle silicates $[25,48,45,33,42]$. For all models a plane wave energy cutoff was set at $100 \mathrm{Ry}$, and a $2 \times 2 \times 2 \mathrm{k}$-space mesh was used, and were tested for convergence. For brg we designed a 2x2x1 supercell, and for ppv we designed a 4x1x1 supercell, each containing 80 atoms in the defect free models. Each structure was relaxed at 0, 30, 60, 90, 100, 110, 120, 130, and $150 \mathrm{GPa}$, and were considered relaxed when the average force per atom was less than $10^{-4}$ $\mathrm{eV} /$ atom. In order to adequately sample the vibrational properties throughout reciprocal space, phonon dispersion calculations were performed on the relaxed structures using a 2x2x2 q-point grid and the resulting force constant matrix was used to interpolate the phonon frequencies on a fine mesh of 260 q-points [3]. Increasing the number of q-points did not significantly affect the free energies of the structures.

\subsection{Quasi-harmonic free-energy calculations}

The Gibbs free-energy $G$ at pressure $P$ and temperature $T$ can be expressed as a minimization problem in the volume $V$ [41]:

$$
G(P, T)=\min _{V}\left\{U^{\text {static }}(V)+F^{v i b}(T ; V)+P V\right\}
$$


where $U^{\text {static }}(V)$ is the energy of the static lattice at volume $V$, and under the quasi-harmonic approximation the vibrational contribution to the free energy $F^{v i b}(V, T)$ is given by:

$$
F^{v i b}(T ; V)=\sum_{s, \mathbf{q}} \frac{1}{2} \hbar \omega_{s}(\mathbf{q} ; V)+k_{B} T \ln \left(1-\exp \left(-\frac{\hbar \omega_{s}(\mathbf{q} ; V)}{k_{B} T}\right)\right)
$$

where the sum runs over all phonon branches, $s$, and all points in the Brillouin zone, $\mathbf{q}$, and $\omega_{s}(\mathbf{q} ; V)$ is the frequency of the $s^{\text {th }}$ phonon at $\mathbf{q}$ at volume $V$, and $\hbar$ is the reduced Planck's constant. To account for the volume dependence of each phonon frequency the set of phonon dispersion curves was used to compute the mode Grüneisen parameters:

$$
\gamma_{s}(\mathbf{q} ; V)=-\frac{V}{\omega_{s}(\mathbf{q} ; V)} \frac{d \omega_{s}(\mathbf{q} ; V)}{d V}
$$

where the derivative is evaluated from finite differences.

In practice, eq. (2) is evaluated for each model by the following scheme: First, the $\left\{\gamma_{s}(\mathbf{q} ; V)\right\}$ 's were computed according to eq. (4). Then for a given pressure and temperature $G(P, T ; V)$ was constructed from a set of isothermal calculations at several volumes in which the $\gamma$ 's were used to adjust the frequencies. Finally, the resulting $V, G$ data were fit with an isothermal equation of state. For the static EOS and the isothermal Gibbs free energy we used the $3^{\text {rd }}$ order Birch-Murnaghan isothermal equation of state:

$U(V)=U_{0}+\frac{9 K_{0 T} V_{0 T}}{16}\left\{K_{0 T}^{\prime}\left[\left(\frac{V_{0 T}}{V}\right)^{2 / 3}-1\right]^{3}+\left[\left(\frac{V_{0 T}}{V}\right)^{2 / 3}-1\right]^{2}\left[6-4\left(\frac{V_{0 T}}{V}\right)^{2 / 3}\right]\right\}$

where $U_{0}$ is the energy at volume $V_{0 T}, K_{0 T}$ is the zero-pressure bulk modulus, and $K_{0 T}^{\prime}$ is the zero-pressure pressure derivative of the bulk modulus, all at constant temperature $T$. 


\section{Results}

\subsection{Defect structures}

The crystal structure models used in this study are shown in fig. 1, and are provided in the supplementary materials. We considered two types of charge-balanced hydrogen defects in both bridgmanite and postperovskite. In the first set of models (hbrg/hppv) we used the $\mathrm{Mg}-2 \mathrm{H}$ defect (1.14 wt.\% water), following Townsend et al. [42]. In the second set of models (Alhbrg/Al-hppv) we used the Si-AlH defect (0.56 wt.\% water). Our hbrg defect structure is somewhat different than the one given in Hernández et al. [19].

The hydrogen bond geometry for all the defect structures at $120 \mathrm{GPa}$ is given in table 1. Over the entire pressure range covered in this study (0-150 $\mathrm{GPa}$ ), the $\mathrm{O}-\mathrm{H}$ bonds for the Al-bearing defect structures of both phases are relatively longer than for the Al-free defects. Upon compression from 0 to $120 \mathrm{GPa}$ the $\mathrm{O}-\mathrm{H}$ bond lengthens for the Al-bearing defect structures by approximately $0.034 \AA$, or $2 \%$, for both structures, and for the $\mathrm{Al}$-free defect structures the $\mathrm{O}-\mathrm{H}$ bonds lengthen by approximately $0.009 \AA$, or $1 \%$. In Alfree hbrg and hppv two hydrogen atoms share a magnesium vacancy, while in Al-hbrg and Al-hppv the hydrogen occupies an interstitial site. Referring to table 1, the hydrogen bonds in Al-free hbrg and hppv are highly asymmetric and non-linear, with a hydrogen bond angle of around 115 degrees. The hydrogen bond in Al-free hppv is slightly stronger, with a hydrogen-bond distance $\mathrm{d}(\mathrm{O} \ldots \mathrm{O})$ about $4 \%$ shorter than in Al-free hbrg. In the Al-bearing system, the hydrogen bonds are considerably more symmetric and linear, with hydrogen bond angles close to 180 degrees. The hydrogen bond in Alhppv is the strongest among all defect models studied here, being about $3 \%$ 
Table 1: Hydrogen bond geometries at $120 \mathrm{GPa}$ for bridgmanite and postperovskite models used in this study.

\begin{tabular}{l||c|c|c|c} 
Defect structure & $d(\mathrm{OH})$ & $d(\mathrm{O} \ldots \mathrm{O})$ & $d(\mathrm{O} \ldots \mathrm{H})$ & H-bond angle \\
& $\AA$ & $\AA$ & $\AA$ & $\mathrm{deg}$. \\
\hline hbrg & 0.996 & 2.397 & 1.800 & 114.94 \\
hppv & 0.993 & 2.307 & 1.708 & 114.73 \\
Al-hbrg & 1.073 & 2.330 & 1.258 & 177.89 \\
Al-hppv & 1.049 & 2.269 & 1.221 & 176.97
\end{tabular}

166 shorter than the hydrogen bond in Al-hbrg and 5\% shorter than the hydrogen 167 bond in Al-free hbrg. 


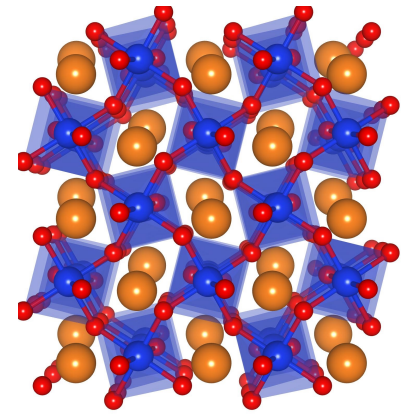

brg

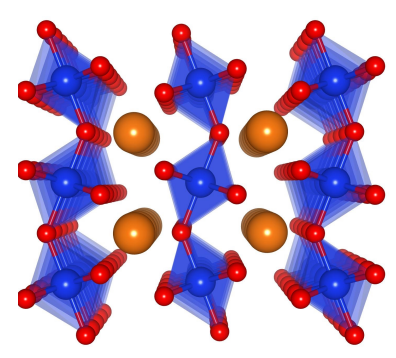

ppv
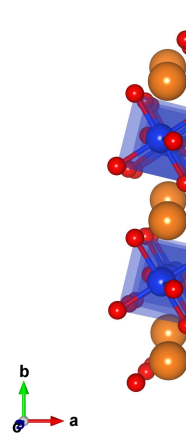

hbrg

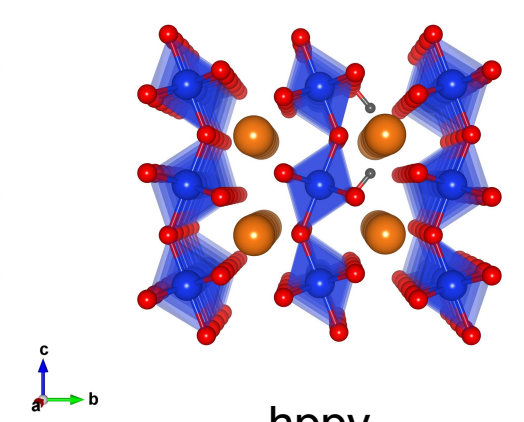

$\varliminf_{a \rightarrow b}^{c}$

hppv

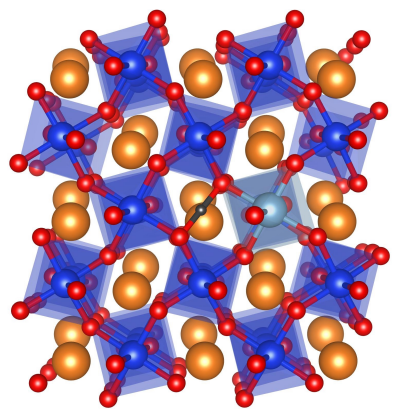

Al-hbrg

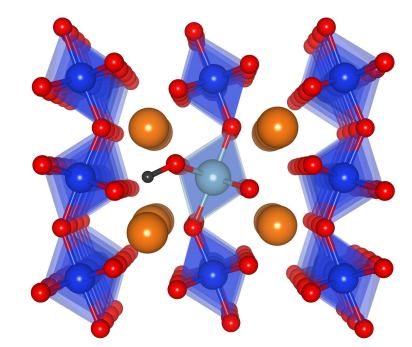

Al-hppv

Figure 1: Crystal structures used in this study. (top left) Al-free bridgmanite, (top middle) Al-free hydrous bridgmanite, (top right) Al-bearing hydrous burdgmanite, (bottom left) Al-free post-perovskite, (bottom middle) Al-free hydrous post-perovskite, and (bottom right) Al-bearing hydrous postperovskite. In all structures orange, dark blue, light blue, red, and black spheres represent magnesium, silicon, aluminum, oxygen, and hydrogen, respectively.

The effect of the defects on the static enthalpy and the defect formation enthalpies for both defects at $0 \mathrm{~K}$ are shown in fig. 2. We find that SiAlH defects favor the ppv phase under static conditions, in agreement with previous DFT calculations [1], while $\mathrm{Mg}-2 \mathrm{H}$ defects favor brg over ppv. The preference for Si-AlH defects in the ppv phase may be due to the difference in 
the bonding environment of hydrogen discussed above rather than aluminum because in ppv and brg at $120 \mathrm{GPa}$ the polyhedral volume of $\mathrm{AlO}_{6}$ is about $3 \%$ larger in ppv compared to brg, consistent with [46]. If we assume the brg-ppv phase boundary at $0 \mathrm{~K}$ depends linearly on the defect concentration, then the $\mathrm{Mg}-2 \mathrm{H}$ defects increase the transition by $8.6 \mathrm{GPa}$ per wt.\% $\mathrm{H}_{2} \mathrm{O}$, whereas for the Si-AlH defects the transition decreases by 11.8 GPa per wt.\% $\mathrm{H}_{2} \mathrm{O}$. From the static enthalpies alone we predict that water should partition into brg over ppv in the absence of aluminum, and into ppv over brg in the presence of aluminum.
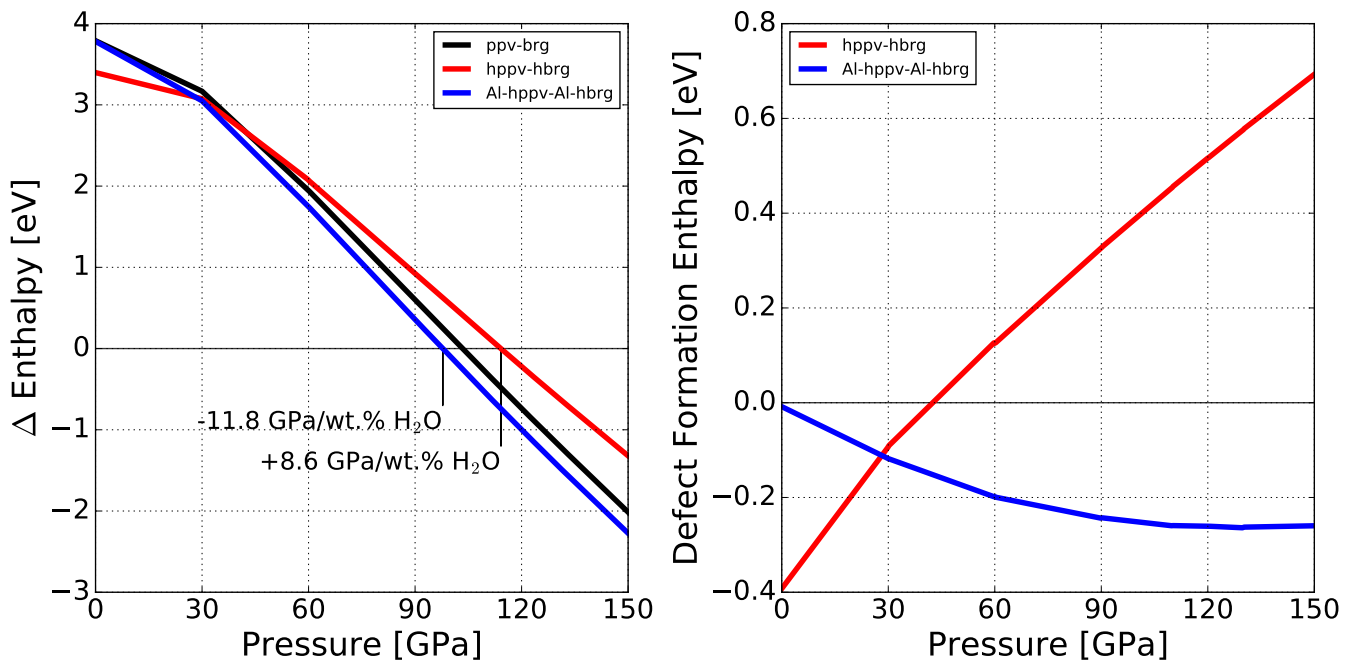

Figure 2: Left: Difference in enthalpy for pure ppv-brg (black), $\mathrm{Mg}-2 \mathrm{H}$ defect models (hppv-hbrg) (red), and Si-AlH defect models (Al-hppv-Al-hbrg) (blue). The dry ppv-brg transition pressure is $104.4 \mathrm{GPa}$, the aluminum-free hppv-hbrg transition pressure is 114.2 $\mathrm{GPa}$, and the Al-hppv-Al-hbrg transition pressure is $97.8 \mathrm{GPa}$. Right: Difference in defect formation enthalpy for Al-free (red) and Al-bearing (blue) hydrogen defects. 
Table 2: $3^{\text {rd }}$ order Birch-Murnaghan equation of state parameters used in this study.

\begin{tabular}{l||c|c|c|c} 
Structure & $K_{0}$ & $K_{0}^{\prime}$ & $V_{0}$ & $U_{0}$ \\
& $\mathrm{GPa}$ & - & $\AA^{3} /$ supercell & $\mathrm{eV} /$ supercell \\
\hline brg & 246 & 3.80 & 662.087 & -23524.565 \\
ppv & 216 & 4.13 & 662.537 & -23520.775 \\
hbrg & 238 & 3.81 & 662.812 & -23523.679 \\
hppv & 205 & 4.17 & 665.543 & -23520.282 \\
Al-hbrg & 239 & 3.82 & 670.251 & -23471.435 \\
Al-hppv & 207 & 4.19 & 670.407 & -23467.653
\end{tabular}

\subsection{Equations of state and free energy calculations}

The static volume-energy curves were used to fit isothermal $3^{\text {rd }}$ order Birch-Murnaghan equations of state (eq. (5)) for each model with a non-linear least squares algorithm and the EOS parameters used for each structure are shown in table 2. Consistent with other nominally anhydrous minerals such as wadsleyite and ringwoodite [9], hydrogen lowers the zero-pressure bulk modulus, increases the zero-pressure volume, and we find a weak dependence of $K_{0}^{\prime}$ on water concentration for all hydrous structures. For both brg and ppv structures the $\mathrm{Mg}-2 \mathrm{H}$ defect models are more compressible than those with $\mathrm{Si}-\mathrm{AlH}$ defects, consistent with the fact that the $\mathrm{Mg}-2 \mathrm{H}$ defect mechanism features hydrogen occupying an Mg-vacancy.

We calculated the phase diagram for dry brg and ppv from the quasiharmonic free energy calculations, presented in fig. 3, as the first step towards computing the partition coefficients. At $125 \mathrm{GPa}$ and $2880 \mathrm{~K}$, we predict a clapeyron slope of 7.6 MPa/K, in good agreement with both experimental 
197 and computational work [36, 34, 48, 31, 20]. Because the QHA assumes 198 the phonon frequencies are temperature independent, calculations at high 199 temperature are not accurate. However, previous studies of QHA energies of 200 brg and ppv indicate that the QHA is valid for brg and ppv in the lowermost 201 mantle [47]. 


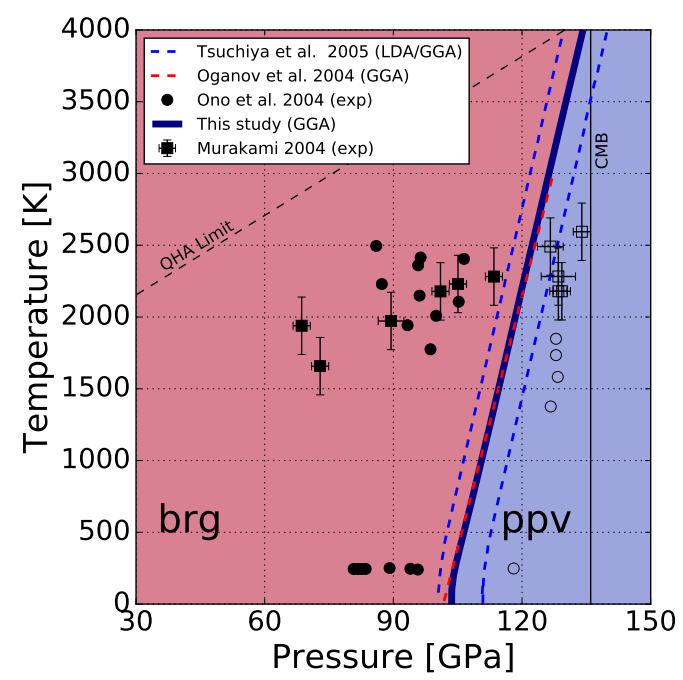

Figure 3: Calculated phase diagram of $\mathrm{MgSiO}_{3}$. Filled and open points represent experimental observation of brg and ppv, respectively [36, 31]. The thin black dashed line shows the limit of the validity of our QHA calculations. Thin colored dashed lines represent phase boundaries from previous first-principles calculations using both the LDA and GGA $[48,34]$. The vertical solid black line denotes the pressure at the core mantle boundary $(\mathrm{CMB})$. 


\subsection{Partition coefficients}

From the brg-ppv phase diagram we compute the partition coefficients between hppv-hbrg and and Al-hppv-Al-hbrg in fig. 4. For the Al-free (Mg$2 \mathrm{H}$ ) system the water partition coefficient between hppv and hbrg (fig. 4 top left) is less than one at every point along the phase boundary, indicating that in equilibrium there are more $\mathrm{Mg}-2 \mathrm{H}$ defects in bridgmanite than postperovskite. Additionally, partitioning along mantle and slab geotherms are not significantly different. At $121.5 \mathrm{GPa}$ and $2400 \mathrm{~K}$, where the mantle geotherm intersects the phase boundary, the partition coefficient is approximately $0.2: 1$, while at $113.5 \mathrm{GPa}$ and $1370 \mathrm{~K}$, where the slab geotherm intersects the phase boundary, the partition coefficient is approximately 0.03:1 (fig. 4 top right). In contrast, in the Al-bearing ( $\mathrm{Si}-\mathrm{AlH}$ ) system the water partition coefficient between ppv and brg is greater than one everywhere, especially at low temperatures (or, equivalently, if traveling along the phase boundary, low pressure) (fig. 4 bottom left). The partitioning of Si-AlH defects is more sensitive to temperature and less sensitive to pressure, which results in more than a factor of two difference in partitioning along ambient mantle and slab geotherms. At $121.5 \mathrm{GPa}$ and $2400 \mathrm{~K}$, where the mantle geotherm intersects the phase boundary, the Al-hppv:Al-hbrg partition coefficient is approximately $3.2: 1$, while at $113.5 \mathrm{GPa}$ and $1370 \mathrm{~K}$, where the slab geotherm intersects the phase boundary, the partition coefficient is approximately 8.1:1 (fig. 4 bottom right). By construction, the partition coefficient requires that brg-ppv are in equilibrium which is true for each pressure only at a single temperature because the brg-ppv phase boundary is univariant for pure $\mathrm{MgSiO}_{3}$. Nevertheless, the effect of temperature on the free energy is 
relatively small compared to the effect of pressure and so the partition coefficient should be valid for small thermal variations about the phase boundary [19]. Therefore the map view of the partition coefficient (fig. 4 left column) is not valid far from the brg-ppv phase boundary, but in both cases it illustrates the general feature that the partition coefficients for both defects are more sensitive to temperature than pressure because the formation energies of the defects are nearly constant as a function of pressure.

\section{Discussion}

Our results suggest that aluminum affects the qualitative behavior of water partitioning between brg and ppv. We estimate that for the hbrg-hppv system the $\mathrm{H}_{2} \mathrm{O}$ partition coefficient is $0.20: 1$ at $121.5 \mathrm{GPa}$ and $2400 \mathrm{~K}$, where the mantle geotherm intersects the phase boundary, and 0.03 at $113.5 \mathrm{GPa}$ and $1370 \mathrm{~K}$, where the slab geotherm intersects the phase boundary. However for the Al-hppv-Al-hbrg system the $\mathrm{H}_{2} \mathrm{O}$ partition coefficient is 3.2:1 at 121.5 GPa and $2400 \mathrm{~K}$, where the mantle geotherm intersects the phase boundary, and 8.1:1 at $113.5 \mathrm{GPa}$ and $1370 \mathrm{~K}$, where the slab geotherm intersects the phase boundary.

The water storage capacity of bridgmanite and partitioning of water between bridgmanite and other minerals has been investigated in several previous studies. Bolfan-Casanova et al. [6] found in the Fe and Al-bearing system, no more than a few ppm of $\mathrm{H}_{2} \mathrm{O}$ was incorporated into bridgmanite with up to about $75 \mathrm{ppm} \mathrm{H}_{2} \mathrm{O}$ in ferropericlase. Murakami et al. [32] found in bridgmanite synthesized from natural hornblende, that bridgmanite contained 0.2 wt.\% water and 6 wt.\% Al, however, their FTIR spectra did 
not resemble $\mathrm{OH}$ in bridgmanite and displayed a strong $3700 \mathrm{~cm}^{-1}$ peak due to brucite, indicative of a hydrous melt phase [39]. Inoue et al. [24] measured the partition coefficient of water in ringwoodite relative to bridgmanite finding 15:1, and estimate 0.1 wt.\% water in bridgmanite synthesized from a starting composition of Fo80 + 16 wt.\% water. Hérnandez et al. [19], using first-principles calculations, computed the $\mathrm{H}_{2} \mathrm{O}$ partition coefficient between pure $\mathrm{MgSi}_{2} \mathrm{O}_{4}$ ringwoodite, $\mathrm{MgO}$ periclase, and $\mathrm{MgSiO}_{3}$ bridgmanite, and found a ringwoodite-bridgmanite $\mathrm{H}_{2} \mathrm{O}$ partition coefficient of about 10:1. Panero et al. [37] synthesized bridgmanite from a natural Fe-bearing (0.1 wt\% Al) hydrous enstatite and found a maximum of $220 \mathrm{ppm}$ water, and complementary first-principles calculations under static conditions confirm a low water partition coefficient for Fe-bearing bridgmanite. 


\subsection{Prospects for seismic detectability}

To illustrate the influence of hydration on the brg-ppv transition on seismic parameters in $\mathrm{D}^{\prime \prime}$, fig. 5 shows the adiabatic bulk sound velocity $\left(V_{\Phi}=\sqrt{K_{S} / \rho}\right)$ for a hypothetical scenario in which we partition 1 wt.\% water between ppv and brg along a $1600 \mathrm{~K}$ isentrope. For a dry composition, a drop in bulk sound velocity of around $-0.09 \mathrm{~km} / \mathrm{s}(-0.08 \%)$ is expected across the brg-ppv phase transition. For a hypothetical hydrous composition assuming 1 wt.\% water and a ppv:brg water partition coefficient of 3.2:1 (i.e. assuming $\mathrm{Si}-\mathrm{AlH}$ defect partitioning), the change in $V_{\Phi}$ is about -0.06 $\mathrm{km} / \mathrm{s}(-0.05 \%)$, or about half that of the dry scenario. At $\mathrm{D}^{\prime \prime}$ pressures it is perhaps unsurprising that the effect of hydration on the velocity is small, however we find that the average velocity gradient $\left(d V_{\Phi} / d P\right)_{S}$ of Al-hppv increases with water content. Additionally, the seismic impedance contrast $\left(\rho_{b r g} V_{\Phi, b r g}-\rho_{p p v} V_{\Phi, p p v}\right)$ in this hydrous scenario is reduced by approximately $50 \%$ of that in the dry scenario. In combination, these two observables might provide a way to probe the hydration state of the lower mantle, as has recently been proposed for the transition zone [40].

\subsection{Limitations}

There are several limitations to our approach which highlight the need for future experiments and simulations. Most importantly, the partition coefficient depends on the static and vibrational energies for a particular defect configuration. Figure 2 indicates that at $\mathrm{D}^{\prime \prime}$ pressures the aluminum-free hydrous defect formation enthalpy is very large and positive, and that the aluminum-bearing hydrous defect formation enthalpy is relatively small and negative. While the exact value of the partition coefficient will change for 
different defect configurations, the overall result, that water partitions preferentially into postperovskite in the presence of aluminum, and preferentially into bridgmanite in the absence of aluminum, is likely robust. Indeed, the stabilization of Al-hppv relative to Al-hbrg was first noticed under static conditions by Akber-Knutson et al. [1]. It is important to point out that the water-partitioning estimates presented here require all of the $\mathrm{Al}$ to be in the form of Si-AlH defects, and as a result may overestimate the partition coefficient. Further, in this study our partition coefficient results assume only a single type of hydrogen defect within each phase, whereas in real crystals there are likely several coexisting varieties of hydrous defects, which should be explored in future studies.

Another important limitation is that we have not accounted for the influence of iron or other minerals on the partitioning. The spin-state of iron in both brg and ppv are still areas of active research, but previous studies indicated that for both brg and ppv iron may adopt an intermediate spin configuration [23]. As both the valence and spin-state of iron affect its ionic radius, the presence of iron will likely have an influence on the partition coefficients. Ferropericlase (fp) coexists with brg and ppv throughout $\mathrm{D}^{\prime \prime}$, and while not a significant host for water itself, would affect the iron partitioning between brg and ppv, and therefore may also affect water partitioning between other phases.[2].

\subsection{Summary}

In summary, our results indicate that aluminous postperovskite has the potential to hold significantly more water than bridgmanite. Recent geochemical measurements suggest the presence of a long-lived primordial reser- 
voir in the deep lower mantle [17]. If geochemically primordial and relatively volatile-rich material exists in $\mathrm{D}^{\prime \prime}$, our results suggest that aluminous ppv may be a host for such primordial water. In the past, melting at the base of the mantle has been proposed to be the last vestiges of a basal magma ocean [28]. However, in regions where primordial hydrous ppv is swept by mantle convection currents through the ppv-brg phase boundary and transforms to brg, the large contrast in the partition coefficient may be an additional mechanism for generating melts via dehydration melting.

\section{Acknowledgements}

JPT was supported by a Presidential Fellowship from Northwestern University and by the EAPSI Program of the U.S. National Science Foundation (NSF) Grant Number 1209633, the Japan Society for the Promotion of Science, and by the Premier Research Institute for Ultrahigh-pressure Sciences (PRIUS) joint research program at the Geodynamics Research Center, Ehime University. This research was also supported in part by NSF grants EAR1452344 (SDJ), EAR-0847951 (CRB), the Carnegie/DOE Alliance Center (CDAC), the David and Lucile Packard Foundation, and by the Alexander Von Humboldt Foundation. Computational resources and staff contributions provided for the Quest high performance computing facility at Northwestern University which is jointly supported by the Office of the Provost, the Office for Research, and Northwestern University Information Technology. Use of the Center for Nanoscale Materials, Argonne National Laboratory, an Office of Science user facility, was supported by the U. S. Department of Energy, Office of Science, Office of Basic Energy Sciences, under Contract No. DE- 
337 AC02-06CH11357. JPT thanks B Tejerina for many fruitful conversations. 

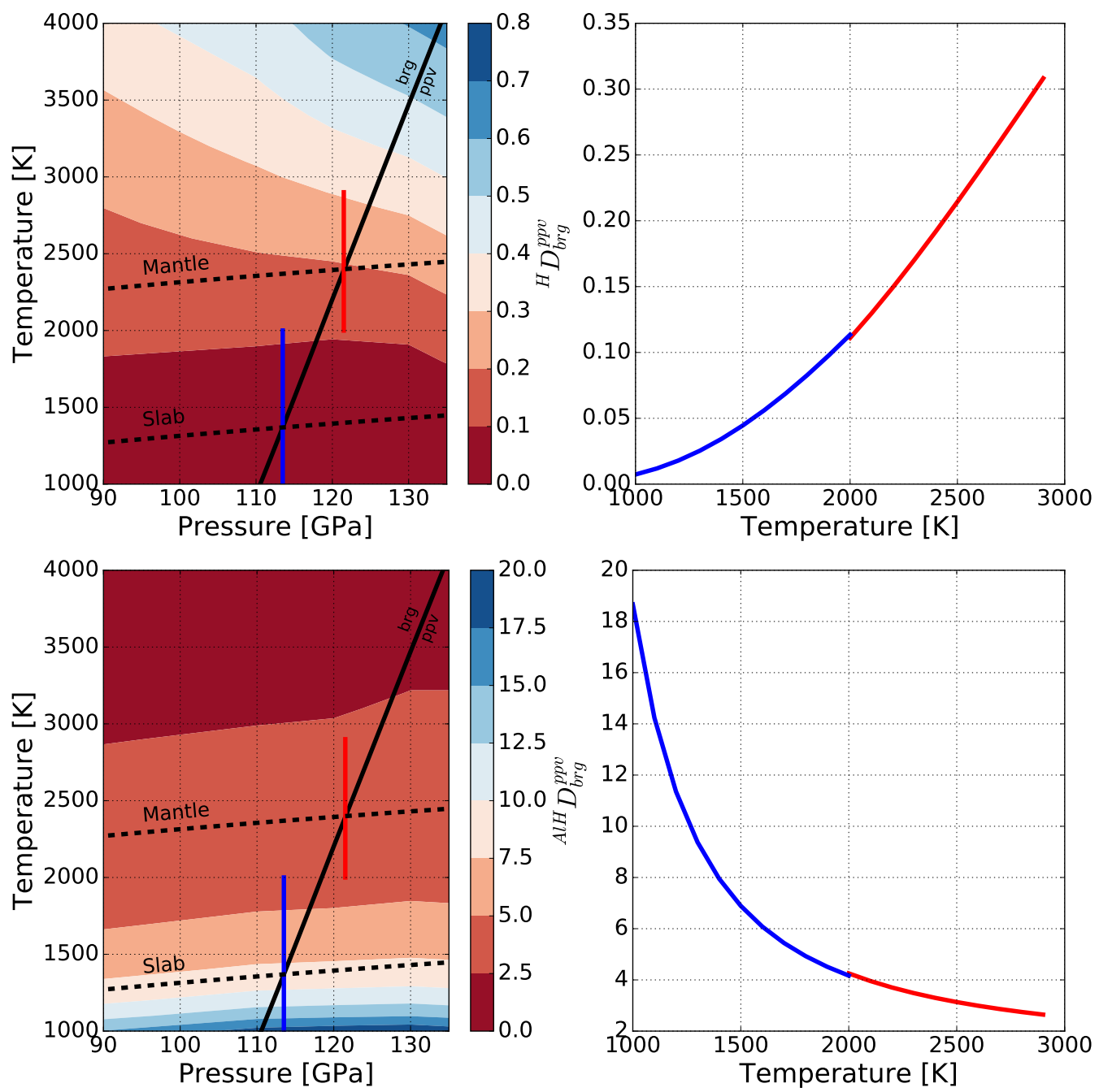

Figure 4: Top left: Map of the $\mathrm{H}_{2} \mathrm{O}$ partition coefficient of ppv relative to brg for the $\mathrm{Mg}-2 \mathrm{H}$ defect model $\left({ }^{H} D_{b r g}^{p p v}\right)$. Top right: Partition coefficients at the brg-ppv coexistence pressures for the mantle (red) and slab (blue). Bottom left: Map of the $\mathrm{H}_{2} \mathrm{O}$ partition coefficient of ppv relative to brg for the Si-AlH defect $\left({ }^{A l H} D_{b r g}^{p p v}\right)$. Bottom right: Partition coefficients at the brg-ppv coexistence pressures for the mantle (red) and slab (blue). In both maps the solid black line is the phase boundary between pure brg-ppv shown in fig. 3, the dotted black lines are mantle and slab adiabats [7,33], and the bright red and blue lines mark the $P, T$ paths of the $1 \mathrm{~d}$ slices shown at right. 


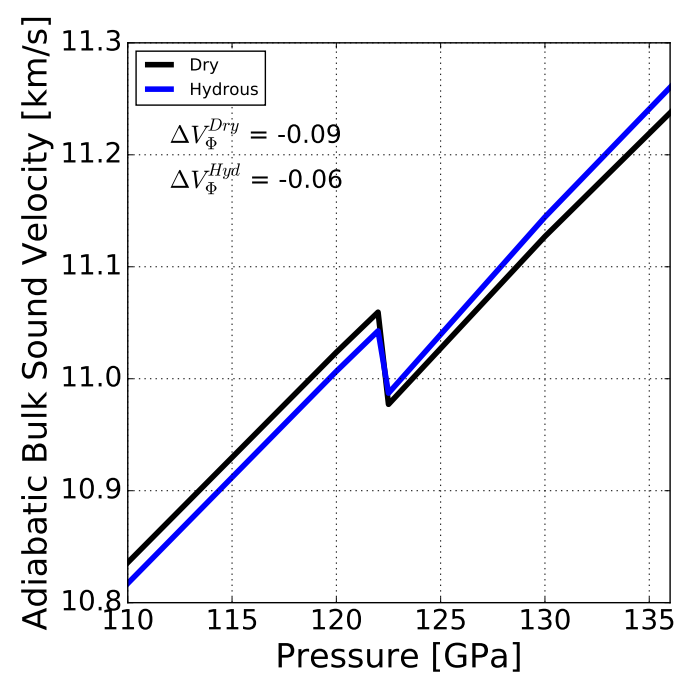

Figure 5: Adiabatic bulk sound velocity along a self-consistently calculated $1600 \mathrm{~K}$ isentrope, for which the brg-ppv transition occurs at $122 \mathrm{GPa}$ and $2516 \mathrm{~K}$. The black curve shows the bulk sound velocity for the dry system, and the blue curve shows the bulk sound velocity for an aluminous hydrous system with an arbitrarily chosen 1 wt.\% water partitioned between ppv and brg assuming a partition coefficient of 3.2:1. 


\section{Appendix A. Derivation of the partition coefficient}

The equilibrium distribution of a dilute component between two otherwise pure bulk solids is determined by the Gibbs free energies of the pure and dilute phases. This derivation draws from that given in [19]. Given a collection of $N$ unit cells of two pure phases $\alpha$ and $\beta$, and $n_{\alpha}$ unit cells of $\alpha$ which contains a single defect, and similarly for $\beta$, where $N>>n_{\alpha}, n_{\beta}$, the Gibbs free energy $G$ of a mixture of pure and defective unit cells of phase $\alpha$ can be written as:

$$
G_{\alpha}\left(n_{\alpha}\right)=G_{\alpha}^{0}\left(n_{\alpha}\right)-T S_{\alpha}^{c o n f}\left(n_{\alpha}\right)
$$

where $T$ is temperature, and $S$ is entropy. The leading term is just the Gibbs free energy of $N$ unit cells composed of $n_{\alpha}$ defect cells and $N-n_{\alpha}$ pure unit cells:

$$
G_{\alpha}^{0}=\left(N-n_{\alpha}\right) F_{\alpha}^{\text {pure }}+n_{\alpha} F_{\alpha}^{\text {defect }}+n_{\alpha} P \Delta V_{\alpha}
$$

where $F$ is the Helmholtz free energy, $P$ is the pressure, and $V$ is the volume. The configurational entropy, $S^{\text {conf }}$ is:

$$
S^{\text {conf }}\left(n_{\alpha}\right)=k_{B} \ln \left(\frac{N_{x} !}{\left(N_{x}-n_{\alpha}\right) ! n_{\alpha} !}\right)
$$

Where $N_{x}$ is the number of possible crystallographic sites of the defect, and $n_{a}$ is the number of defects. To see the effect of $n_{\alpha}$ on the total energy we compute the partial molar derivative of eq. (A.1):

$$
\frac{\partial G_{\alpha}\left(n_{\alpha}\right)}{\partial n_{\alpha}}=-F_{\alpha}^{\text {pure }}+F_{\alpha}^{\text {defect }}-T \frac{\partial S_{\alpha}^{\text {conf }}\left(n_{\alpha}\right)}{\partial n_{\alpha}}+P \Delta V_{\alpha}=G_{\alpha}^{f}-T \frac{\partial S_{\alpha}^{\text {conf }}\left(n_{\alpha}\right)}{\partial n_{\alpha}}
$$

where $G_{\alpha}^{f}=G^{\text {defect }}-G^{\text {pure }}$, is the Gibbs free energy of formation. The partial molar derivative of the configurational entropy is:

$$
\frac{\partial S_{\alpha}^{c o n f}}{\partial n_{\alpha}}=k_{B} \ln \left(\frac{N_{x}-n_{\alpha}}{n_{\alpha}}\right)
$$


356

357

$\frac{\partial G_{\beta}}{\partial n_{\beta}}=G_{\beta}^{f}-k_{B} T \ln \left(\frac{N_{x}-n_{\beta}}{n_{\beta}}\right)$

Setting both derivatives equal to zero at equilibrium:

$$
\begin{aligned}
\frac{G_{\alpha}^{f}}{k_{B} T}=\ln \left(\frac{N_{x}-n_{\alpha}}{n_{\alpha}}\right) \Longrightarrow e^{G_{\alpha}^{f} / k_{B} T}=\frac{N_{x}-n_{\alpha}}{n_{\alpha}} \\
\frac{G_{\beta}^{f}}{k_{B} T}=\ln \left(\frac{N_{x}-n_{\beta}}{n_{\beta}}\right) \Longrightarrow e^{G_{\beta}^{f} / k_{B} T}=\frac{N_{x}-n_{\beta}}{n_{\beta}}
\end{aligned}
$$

Solving for $n_{\alpha}$ and $n_{\beta}$ we obtain the pair of equations:

$$
\begin{aligned}
& n_{\alpha}=\frac{N_{x}}{e^{G_{\alpha}^{f} / k_{B} T}+1} \\
& n_{\beta}=\frac{N_{x}}{e^{G_{\beta}^{f} / k_{B} T}+1}
\end{aligned}
$$

Finally, the partition coefficient $\left(D_{\beta}^{\alpha}\right)$, is just the ratio of the number of defects in the two phases:

$$
D_{\beta}^{\alpha}=\frac{n_{\alpha}}{n_{\beta}}=\frac{e^{G_{\beta}^{f} / k_{B} T}+1}{e^{G_{\alpha}^{f} / k_{B} T}+1} \approx e^{\Delta G_{\beta-\alpha}^{f} / k_{B} T}
$$

The above derivation assumes equal amounts $N$ of $\alpha$ and $\beta$, the only conditions under which such can coexist is along the phase boundary. As a result, it should be understood that this formulation is accurate only along the phase boundary and only for dilute concentrations.

An alternative derivation works directly from the chemical potentials. For two components -1 , the dilute solute, and 2 , the solvent - and for two phases $-\alpha$ and $\beta$, each composed of components 1 and 2 - the conditions of exact 
equilibrium are:

$$
\begin{aligned}
& \mu_{1}^{\alpha}\left(P, T, X_{1}^{\alpha}\right)=\mu_{1}^{\beta}\left(P, T, X_{1}^{\beta}\right) \\
& \mu_{2}^{\alpha}\left(P, T, X_{2}^{\alpha}\right)=\mu_{2}^{\beta}\left(P, T, X_{2}^{\beta}\right)
\end{aligned}
$$

where $\mu_{1}^{\alpha}$ is the chemical potential of component 1 in phase $\alpha$, and similarly for the other terms. Assuming an ideal solution, the $\mu$ 's take the following form:

$$
\begin{gathered}
{ }^{0} \mu_{1}^{\alpha}(P, T)+k_{B} T \ln X_{1}^{\alpha}={ }^{0} \mu_{1}^{\beta}(P, T)+k_{B} T \ln X_{1}^{\beta} \\
{ }^{0} \mu_{2}^{\alpha}(P, T)+k_{B} T \ln \left(1-X_{1}^{\alpha}\right)={ }^{0} \mu_{2}^{\beta}(P, T)+k_{B} T \ln \left(1-X_{1}^{\beta}\right)
\end{gathered}
$$

with $1=X_{1}^{\alpha}+X_{2}^{\alpha}$ and likewise for $\beta$, and the superscript 0 denotes the chemical potential for the pure end-member. Rearranging for $X_{1}$ in both phases we obtain:

$$
\begin{aligned}
& X_{1}^{\alpha}=X_{1}^{\beta} e^{\Delta^{0} \mu_{1}^{\beta-\alpha} / k_{B} T} \\
& X_{1}^{\beta}=\frac{1-e^{\Delta^{0} \mu_{2}^{\beta-\alpha} / k_{B} T}}{e^{\Delta^{0} \mu_{1}^{\beta-\alpha} / k_{B} T}-e^{\Delta^{0} \mu_{2}^{\beta-\alpha} / k_{B} T}}
\end{aligned}
$$

where $\Delta^{0} \mu_{1}^{\beta-\alpha}$ is the difference of the chemical potentials of the two endmembers. Finally, the partition coefficient is just the ratio of the concentrations of the dilute component 1 in both phases:

$$
{ }^{1} D_{\beta}^{\alpha}=\frac{X_{1}^{\alpha}}{X_{1}^{\beta}}=e^{\Delta^{0} \mu_{1}^{\beta-\alpha} / k_{B} T}
$$

To show these two approaches are equivalent, we make the simplification $X_{1}=X$ and $X_{2}=1-X$, and denote by $\tilde{G}$ the free energy per cell, so that the Gibbs free energies of formation are:

$$
\begin{aligned}
& \tilde{G}_{\text {defect }}^{\alpha}-\tilde{G}^{\alpha}=(1-X)^{0} \mu_{2}^{\alpha}+X^{0} \mu_{1}^{\alpha}+k_{B} T(X \ln (X)+(1-X) \ln (1-X))-{ }^{0} \mu_{2}^{\alpha} \\
& \tilde{G}_{\text {defect }}^{\beta}-\tilde{G}^{\beta}=(1-X)^{0} \mu_{2}^{\beta}+X^{0} \mu_{1}^{\beta}+k_{B} T(X \ln (X)+(1-X) \ln (1-X))-{ }^{0} \mu_{2}^{\beta}
\end{aligned}
$$


367 The differential Gibbs free energy of formation is the difference of the above:

$$
\Delta \tilde{G}^{f}=\tilde{G}_{d e f e c t}^{\alpha}-\tilde{G}^{\alpha}-\left(\tilde{G}_{d e f e c t}^{\beta}-\tilde{G}^{\beta}\right)=X\left({ }^{0} \mu_{1}^{\alpha}-{ }^{0} \mu_{2}^{\alpha}-\left({ }^{0} \mu_{1}^{\beta}-{ }^{0} \mu_{2}^{\beta}\right)\right)
$$

${ }_{368}$ But, on the phase boundary ${ }^{0} \mu_{2}^{\alpha} \approx{ }^{0} \mu_{2}^{\beta}$ if the defects are dilute, which leaves:

$$
e^{\Delta \tilde{G}^{f} / k_{B} T}=e^{X \Delta^{0} \mu_{1}^{\alpha-\beta} / k_{B} T}
$$

369 Which is equivalent to the result of Hernández et al. [19] in the case of equal 370 numbers of equivalent positions in each structure. However, if the number 371 of equivalent positions is different in the two phases, then the above must 372 be modified to take into account mixing on sites. Therefore, the partition 373 coefficient can be viewed in terms of standard equilibrium chemical thermo374 dynamics in the limit of dilute ideal mixing. 
[1] Akber-Knutson, S., Steinle-Neumann, G., Asimow, P.D., 2005. Effect of $\mathrm{Al}$ on the sharpness of the $\mathrm{MgSiO}_{3}$ perovskite to postperovskite phase transition. Geophysical Research Letters 32. doi:10.1029/2005GL023192.

[2] Auzende, A.L., Badro, J., Ryerson, F., Weber, P., Fallon, S., Addad, A., Siebert, J., Fiquet, G., 2008. Element partitioning between magnesium silicate perovskite and ferropericlase: New insights into bulk lower-mantle geochemistry. Earth and Planetary Science Letters 269, 164 - 174. doi:http://dx.doi.org/10.1016/j.epsl.2008.02.001.

[3] Baroni, S., de Gironcoli, S., Dal Corso, A., 2001. Phonons and related crystal properties from density-functional perturbation theory. Reviews of Modern Physics 73, 515-562. doi:10.1103/RevModPhys.73.515.

[4] von Barth, U., 1984. Electronic structure of complex materials. NATO Advanced Studies Institutes Series B, Physics 113.

[5] Bercovici, D., Karato, S.I., 2003. Whole-mantle convection and the transition-zone water filter. Nature 425, 39-44. doi:10.1038/nature01918.

[6] Bolfan-Casanova, N., Keppler, H., Rubie, D., 2000. Water partitioning between nominally anhydrous minerals in the $\mathrm{MgO}-\mathrm{SiO}_{2}-\mathrm{H}_{2} \mathrm{O}$ system up to 24 GPa: Implications for the distribution of water in the Earths mantle. Earth and Planetary Science Letters 182, 209-221. doi:10.1016/S0012-821X(00)00244-2. 
[7] Brown, J., Shankland, T., 1981. Thermodynamic parameters in the earth as determined from seismic profiles. Geophysical Journal International 66, 579-596. doi:10.1111/j.1365-246X.1981.tb04891.x.

[8] Bullen, K., 1949. Compressibility-pressure hypothesis and the earth's interior. Geophysical Supplements to the Monthly Notices of the Royal Astronomical Society 5, 335-368. doi:10.1111/j.1365-246X.1949.tb02952.x.

[9] Chang, Y., Jacobsen, S., Bina, C., Thomas, S., Smyth, J., Frost, D., Boffa Ballaran, T., McCammon, C., Hauri, E., Inoue, T., Yurimoto, H., Meng, Y., Dera, P., 2015. Comparative compressibility of hydrous wadsleyite and ringwoodite: Effect of $\mathrm{H}_{2} \mathrm{O}$ and implications for detecting water in the transition zone. Journal of Geophysical Research: Solid Earth 120, 8259-8280. doi:10.1002/2015JB012123.

[10] Cobden, L., Thomas, C., Trampert, J., 2015. Seismic detection of post-perovskite inside the earth, in: Khan, A., Deschamps, F. (Eds.), The Earth's Heterogeneous Mantle. Springer International Publishing. Springer Geophysics, pp. 391-440. doi:10.1007/978-3-319-15627-9_13.

[11] Deschamps, F., Li, Y., Tackley, P., 2015. Large-scale thermochemical structure of the deep mantle: Observations and models, in: Khan, A., Deschamps, F. (Eds.), The Earth's Heterogeneous Mantle. Springer International Publishing. Springer Geophysics, pp. 479-515. doi:10.1007/978-3-319-15627-9_15.

[12] Fiquet, G., Auzende, A.L., Siebert, J., Corgne, A., Bureau, H., Ozawa, 
H., Garbarino, G., 2010. Melting of peridotite to 140 gigapascals. Science 329, 1516-1518. doi:10.1126/science.1192448.

[13] Garnero, E., McNamara, A., 2008. Structure and dynamics of Earth's lower mantle. Science 320, 626-628. doi:10.1126/science.1148028.

[14] Giannozzi, P., et al., 2009. QUANTUM ESPRESSO: A modular and opensource software project for quantum simulations of materials. Journal of Physics: Condensed Matter 21, 395502. doi:10.1088/0953$8984 / 21 / 39 / 395502$.

[15] Gonnermann, H., Mukhopadhyay, S., 2007. Non-equilibrium degassing and a primordial source for helium in ocean-island volcanism. Nature 449, 1037-1040. doi:10.1038/nature06240.

[16] Grocholski, B., Catalli, K., Shim, S.H., Prakapenka, V., 2012. Mineralogical effects on the detectability of the postperovskite boundary. Proceedings of the National Academy of Sciences 109, 2275-2279. doi:10.1073/pnas.1109204109.

[17] Hallis, L.J., Huss, G.R., Nagashima, K., Taylor, G.J., Halldórsson, S.A., Hilton, D.R., Mottl, M.J., Meech, K.J., 2015. Evidence for primordial water in Earths deep mantle. Science 350, 795-797. doi:10.1126/science.aac4834.

[18] Helmberger, D., Lay, T., Ni, S., Gurnis, M., 2005. Deep mantle structure and the postperovskite phase transition. Proceedings of the National Academy of Sciences of the United States of America 102, 17257-17263. doi:10.1073/pnas.0502504102. 
[19] Hernández, E., Alfè, D., Brodholt, J., 2013. The incorporation of water into lower-mantle perovskites: A first-principles study. Earth and Planetary Science Letters 364, 37-43. doi:10.1016/j.epsl.2013.01.005.

[20] Hirose, K., 2006. Postperovskite phase transition and its geophysical implications. Reviews of Geophysics 44. doi:10.1029/2005RG000186.

[21] Hirschmann, M., 2006. Water, melting, and the deep Earth $\mathrm{H}_{2} \mathrm{O}$ cycle. Annual Review of Earth and Planetary Sciences 34, 629-653. doi:10.1146/annurev.earth.34.031405.125211.

[22] Hohenberg, P., Kohn, W., 1964. Inhomogeneous electron gas. Physical Review 136, B864-B871.

[23] Hsu, H., Umemoto, K., Wu, Z., Wentzcovitch, R., 2010. Spin-state crossover of iron in lower-mantle minerals: Results of $\mathrm{DFT}+\mathrm{U}$ investigations. Reviews in Mineralogy \& Geochemistry 71, 169-199. doi:10.2138/rmg.2010.71.09.

[24] Inoue, T., Wada, T., Sasaki, R., Yurimoto, H., 2010. Water partitioning in the Earths mantle. Physics of the Earth and Planetary Interiors 183, 245-251. doi:10.1016/j.pepi.2010.08.003.

[25] Karki, B., Wentzcovitch, R., 2000. Ab initio lattice dynamics of $\mathrm{MgSiO}_{3}$ perovskite at high pressure. Physical Review B 62, 750-756. doi:10.1103/PhysRevB.62.14750.

[26] Kohlstedt, D., Keppler, H., Rubie, D., 1996. Solubility of water in the $\alpha, \beta$ and $\gamma$ phases of $(\mathrm{Mg}, \mathrm{Fe})_{2} \mathrm{SiO}_{4}$. Contributions to Mineralogy and Petrology 123, 345-357. doi:10.1007/s004100050161. 
[27] Kohn, W., Sham, L., 1965. Self-consistent equations including exchange and correlation effects. Physical Review 140, A1133-A1138.

[28] Labrosse, S., Hernlund, J., Coltice, N., 2007. A crystallizing dense magma ocean at the base of the Earths mantle. Nature 450, 866-869. doi:10.1038/nature06355.

[29] Lay, T., Garnero, E., 2011. Deep mantle seismic modeling and imaging. Annual Review of Earth and Planetary Sciences 39, 91-123. doi:10.1146/annurev-earth-040610-133354.

[30] Monkhorst, H., Pack, J., 1976. Special points for Brillouinzone integrations. Physical Review B 13, 5188-5192. doi:10.1103/PhysRevB.13.5188.

[31] Murakami, M., Hirose, K., Kawamura, K., Sata, N., Ohishi, Y., 2004. Post-perovskite phase transition in $\mathrm{MgSiO}_{3}$. Science 304, 855-858. doi:10.1126/science.1095932.

[32] Murakami, M., Hirose, K., Yurimoto, H., Nakashima, S., Takafuji, N., 2002. Water in Earth's lower mantle. Science 295, 1885-1887. doi:10.1126/science.1065998.

[33] Nishi, M., Irifune, T., Tsuchiya, J., Tange, Y., Nishihara, Y., K., F., Higo, Y., 2014. Stability of hydrous silicate at high pressures and water transport to the deep lower mantle. Nature Geoscience 7, 224-227. doi:10.1038/NGEO2074.

[34] Oganov, A., Ono, S., 2004. Theoretical and experimental evidence for 
a post-perovskite phase of $\mathrm{MgSiO}_{3}$ in earth's $\mathrm{D}^{\prime \prime}$ layer. Nature 430, 445-448. doi:10.1038/nature02701.

[35] Ohtani, E., Litasov, K., Hosoya, T., Kubo, T., Kondo, T., 2004. Water transport into the deep mantle and formation of a hydrous transition zone. Physics of the Earth and Planetary Interiors 143144, 255-269. doi:10.1016/j.pepi.2003.09.015.

[36] Ono, S., Ohishi, Y., Mibe, K., 2004. Phase transition of Ca-perovskite and stability of Al-bearing Mg-perovskite in the lower mantle. American Mineralogist 89, 1480-1485. doi:10.2138/am-2004-1016.

[37] Panero, W.R., Pigott, J.S., Reaman, D.M., Kabbes, J.E., Liu, Z., 2015. Dry (mg,fe)sio3 perovskite in the earth's lower mantle. Journal of Geophysical Research: Solid Earth 120, 894-908. doi:10.1002/2014JB011397.

[38] Perdew, J., Burke, K., Wang, Y., 1996. Generalized gradient approximation for the exchange-correlation hole of a many-electron system. Physical Review B 54, 16533-16539. doi:10.1103/PhysRevB.54.16533.

[39] Schmandt, B., Jacobsen, S., Becker, T., Liu, Z., Ducker, K., 2014. Dehydration melting at the top of the lower mantle. Science 344, 1265-1268. doi:10.1126/science.1253358.

[40] Thio, V., Cobden, L., Trampert, J., 2016. Seismic signature of a hydrous mantle transition zone. Physics of the Earth and Planetary Interiors 250, 46-63. doi:10.1016/j.pepi.2015.11.005. 
[41] Togo, A., Chaput, L., Tanaka, I., Hug, G., 2010. First-principles phonon calculations of thermal expansion in $\mathrm{Ti}_{3} \mathrm{SiC}_{2}, \mathrm{Ti}_{3} \mathrm{AlC}_{2}$, and $\mathrm{Ti}_{3} \mathrm{GeC}_{2}$. Phys. Rev. B 81, 174301. doi:10.1103/PhysRevB.81.174301.

[42] Townsend, J., Tsuchiya, J., Bina, C., Jacobsen, S., 2015. First-principles investigation of hydrous post-perovskite. Physics of the Earth and Planetary Interiors 244, 42-48. doi:10.1016/j.pepi.2015.03.010.

[43] Troullier, N., Martins, J., 1991. Efficient pseudopotentials for plane-wave calculations. Physical Review B 43, 1993-2006. doi:10.1103/PhysRevB.43.1993.

[44] Tsuchiya, J., Tsuchiya, T., 2008. Postperovskite phase equilibria in the $\mathrm{MgSiO}_{3}-\mathrm{AlSiO}_{3}$ system. Proceedings of the National Academy of Sciences 105. doi:10.1073/pnas.0805660105.

[45] Tsuchiya, J., Tsuchiya, T., 2009. First principles investigation of the structural and elastic properties of hydrous wadsleyite under pressure. Journal of Geophysical Research 114, B02206. doi:10.1029/2008JB005841.

[46] Tsuchiya, J., Tsuchiya, T., Wentzcovitch, R., 2005a. Transition from the $\mathrm{rh}_{2} \mathrm{O}_{3}(i i)$-to-CaIro 3 structure and the high-pressuretemperature phase diagram of alumina. Physical Review B 72, 020103. doi:10.1103/PhysRevB.72.020103.

[47] Tsuchiya, J., Tsuchiya, T., Wentzcovitch, R., 2005b. Vibrational and thermodynamic properties of $\mathrm{mgsio}_{3}$ postperovskite. Journal of Geophysical Research 110, B02204. doi:10.1029/2004JB003409. 
532 [48] Tsuchiya, T., Tsuchiya, J., Umemoto, K., Wentzcovitch, R., 2004. Phase 533 transition in $\mathrm{MgSiO}_{3}$ perovskite in the earth's lower mantle. Earth and 534 Planetary Science Letters 224, 241-248. doi:10.1016/j.epsl.2004.05.017. 535 [49] Wicks, J.K., Jackson, J.M., Sturhahn, W., 2010. Very low 536 sound velocities in iron-rich $(\mathrm{Mg}, \mathrm{Fe}) \mathrm{O}$ : Implications for the core537 mantle boundary region. Geophysical Research Letters 37, L15304. 538 doi:10.1029/2010GL043689.

[50] Williams, C.D., Li, M., McNamara, A.K., Garnero, E.J., van Soest, M.C., 2015. Episodic entrainment of deep primordial mantle material into ocean island basalts. Nature communications 6 . doi:10.1038/ncomms9937. 